\title{
Transmissão de fungos via semente e patogenicidade de Pestalotiopsis sp. em mudas de Schinus terebinthifolius Raddi
}

\section{Seed transmission and pathogenicity of Pestalotiopsis sp. in seedlings of Schinus terebinthifolius Raddi}

\author{
Caciara Gonzatto Maciel ${ }^{1 *}$; Tiago Silveira Ferrera ${ }^{2}$; Marciéli Pitorini Bovolini³; \\ Rogério Gonzatto ${ }^{4}$; Marlove Fátima Brião Muniz ${ }^{5}$; Galileo Adeli Buriol ${ }^{6}$
}

Resumo

\begin{abstract}
Este trabalho objetivou verificar a transmissão de fungos via sementes de Schinus terebinthifolius Raddi. (aroeira-vermelha) coletadas no município de Santa Maria - RS, assim como, a patogenicidade do fungo Pestalotiopsis sp. isolado a partir dessas sementes, em mudas de aroeira-vermelha. Para o teste de transmissão foram utilizadas 100 sementes, divididas em quatro repetições de 25 cada. A semeadura foi realizada em areia e as avaliações foram semanais, quantificou-se o número de plântulas emergentes e sintomáticas. O fungo Pestalotiopsis sp. identificado em alto percentual, tanto no teste de sanidade (55\%), quanto no teste de transmissão (52\%), foi isolado em meio de cultura, para posterior verificação da patogenicidade. A inoculação de Pestalotiopsis sp. em mudas de aroeira-vermelha foi realizada via aspersão na concentração de $2.10^{6}$ conídios $/ \mathrm{mL}$, as avaliações foram semanais durante 28 dias. Os fungos identificados no teste de transmissão foram: Pestalotiopsis sp. (52\%), Fusarium sp. (15\%) e Cladosporium sp. (12\%). O fungo Pestalotiopsis sp. apresentou-se patogênico as mudas de aroeira-vermelha, causando manchas foliares, que coalesceram com o passar da tempo e levaram a queda da folhas.
\end{abstract}

Palavras-chave: Aroeira-vermelha, ornamental, germinação, sanidade, fungo

\begin{abstract}
This study aimed to verify the transmission of fungi through seeds of Schinus terebinthifolius Raddi. (aroeira-vermelha) collected in the municipality of Santa Maria-RS, as well as the pathogenicity of the fungus Pestalotiopsis sp. Isolated from these seeds, aroeira-vermelha seedlings. For the transmission test 100 seeds were used, divided into four replicates of 25 each. The seeds were sown in sand and the evaluations were weekly, quantified the number of seedlings emerging and symptomatic. The fungus Pestalotiopsis sp. identified in a high percentage in both the sanity test (55\%) and in the transmission test (52\%) was isolated in culture medium for subsequent verification of pathogenicity. Inoculation of Pestalotiopsi sp. in seedlings of aroeira-vermelha spraying was carried out via the concentration of $2.10^{6}$ conidia/mL, the evaluations were weekly for 28 days. The fungi identified in the test transmission were: Pestalotiopsis sp. (52\%), Fusarium sp. (15\%) and Cladosporium sp. (12\%). The fungus Pestalotiopsis sp. presented the seedlings of aroeira-vermelha pathogenic, causing leaf spots that coalesced with the passage of time, and lead to the fall of leaves.
\end{abstract}

Key words: Aroeira-vermelha, ornamental, germination, sanity, fungus

${ }^{1}$ Enga Florestal. Mestranda do Programa de Pós-Graduação em Engenharia Florestal, Universidade Federal de Santa Maria, UFSM, Santa Maria, RS. E-mail: caciaragonzatto@gmail.com

${ }^{2}$ Biólogo. Mestrando do Programa de Pós-Graduação em Agrobiologia da UFSM, Santa Maria, RS. E-mail: tsferrera@yahoo.com.br

${ }^{3}$ Discente de Engenharia Florestal da UFSM, Santa Maria, RS. E-mail: maninhabovolini@yahoo.com.br

${ }^{4}$ Eng $^{\mathrm{o}}$ Agr ${ }^{\circ}$, Mestrando do Programa de Pós-Graduação em Ciência do Solo da UFSM, Santa Maria, RS. E-mail: rogonzatto@ gmail.com

${ }^{5}$ Prof $^{\mathrm{a}}$. do Dept ${ }^{\mathrm{o}}$ de Defesa Fitossanitária da UFSM, Santa Maria, RS. E-mail: marlovemuniz@yahoo.com.br

${ }^{6}$ Prof. do Dept ${ }^{\circ}$ de Ciências Naturais e Exatas da UFSM, Santa Maria, RS. E-mail: galileoburiol@yahoo.com.br

* Autor para correspondência 


\section{Introdução}

A aroeira-vemelha (Schinus terebenthifolius Raddi.) é uma árvore de pequeno porte que apresenta frutos vermelhos que persistem por longos períodos na planta. Tais características tornam esta planta adequada para uso ornamental assim como para a arborização urbana. Além disso, a S. terebenthifolius é uma importante espécie para a regeneração de mata ripária e de áreas degradadas (LORENZI, 2002).

A utilização de espécies florestais nativas para plantios com finalidade comercial ou ambiental, necessita de estudos para o desenvolvimento de tecnologia adequada de produção, iniciando pelo conhecimento da qualidade das suas sementes (LEONHARDT et al., 2001).

Diversos gêneros fúngicos são encontrados associados a sementes de aroeira-vermelha, tais como, Fusarium, Alternaria, Pestalotia, Penicillium, Aspergillus, Trichoderma, Chaetomium, Nigrospora, Geotrichum e Mucor, e estes quando transmitidos via semente, podem causar danos nas plântulas (STRAPASSON; SANTOS; MEDEIROS, 2002).

O gênero Pestalotiopsis é considerado potencialmente patogênico, em função dos danos relatados, quando associado a sementes ou mudas. Santos, Medeiros e Santana (2001) citam Pestalotiopsis sp. como agente causal de podridões de sementes em condições inadequadas de armazenamento. Esse fitopatógeno foi identificado causando danos em mudas de aroeira-vermelha na região de Piracicaba - SP (BOTELHO, 2006).

A partir de testes prévios de germinação e sanidade em sementes de Schinus terebinthifolius, coletadas no município de Santa Maria - RS no ano de 2011 verificou-se a necessidade de constatar se os fungos associados às sementes eram transmitidos para plântula, e se o fungo Pestalotiopsis sp. apresentava patogenicidade quando inoculado em mudas de Schinus terebinthifolius.

\section{Material e Métodos}

Local dos experimentos e origem das sementes

Os trabalhos foram conduzidos no Laboratório de Pesquisa em Fitopatologia no Departamento de Defesa Fitossanitária da Universidade Federal de Santa Maria (UFSM), Santa Maria-RS. As sementes de aroeira foram coletadas no mês de janeiro, na área do Jardim Botânico nas dependências da UFSM (2942'S e 534' W), no campus Camobi a uma altitude aproximada de $95 \mathrm{~m}$. O clima da região é subtropical úmido, com temperatura média anual de $19,2^{\circ} \mathrm{C}$ e precipitação pluvial média anual de 1708 mm (LEMES; RITTER; MORAIS, 2008).

\section{Teste de germinação e sanidade}

Foram utilizadas 100 sementes para cada teste, germinação e sanidade, divididas em quatro repetições de 25 cada. Para ambos os testes a semeadura foi realizada em caixas "gerbox" previamente desinfestadas (álcool $70 \%$ e hipoclorito de sódio $1 \%$ ) e, forradas com papelfiltro umedecido com água destilada esterilizada. $\mathrm{O}$ material foi acondicionado em um germinador com temperatura de $25^{\circ} \mathrm{C}$ e luz contínua por 24 horas (LAZAROTTO; MUNIZ; SANTOS, 2010).

A avaliação da germinação das sementes foi realizada por meio de contagem de plântulas germinadas 14 dias após a instalação do teste. Aos 30 dias contabilizou-se o número de plântulas emergidas e sementes não germinadas.

A identificação dos fungos presentes nas sementes em nível de gênero foi realizada sete dias após a incubação, com auxílio de microscópios estereoscópico e óptico (BARNETT; HUNTER, 1972). Os resultados foram expressos em percentagem de incidência para cada gênero fúngico. No caso de identificação de algum fungo considerado potencialmente patogênico no teste de sanidade, este foi isolado em meio BatataDextrose-Ágar (BDA: 200g batata - 20g dextrose - 20g de Ágar - $1000 \mathrm{ml}$ de água) suplementado 
com antibióticos (sulfato de estreptomicina), para obtenção da cultura pura e posterior verificação da patogenicidade do mesmo.

\section{Teste de transmissão de fungos via sementes}

Para o teste de transmissão de fungos, foram semeadas 100 sementes divididas em quatro repetições de 25 cada. A semeadura foi realizada em “gerbox' previamente desinfestadas (álcool 70\% e hipoclorito de sódio $1 \%$ ), tendo como substrato areia autoclavada a $121^{\circ} \mathrm{C}$ durante 60 minutos, duas vezes consecutivas, com um intervalo de 24 horas entre cada esterilização. O material foi mantido em sala climatizada a $25{ }^{\circ} \mathrm{C}$ e umedecido sempre que necessário (REGO, 2008).

Avaliou-se a percentagem de emergência e a ocorrência de sintomas nas plântulas, semanalmente durante 28 dias. As plântulas sintomáticas e as sementes não germinadas foram colocadas em câmara úmida para observação de estruturas fúngicas.

Patogenicidade de Pestalotiopsis sp. isolado de sementes de Schinus terebinthifolius em mudas

O isolado de Pestalotiopsis sp. utilizado no teste de patogenicidade foi obtido no teste de sanidade de sementes. O isolamento e cultivo foram realizados em placas de Petri contendo o meio de cultivo BDA. As placas foram mantidas em câmara de incubação a $25 \pm 2{ }^{\circ} \mathrm{C}$ por sete dias.

Posteriormente, foram acrescentados $20 \mathrm{~mL}$ de água esterilizada em cada placa de Petri e procedeuse a raspagem das colônias com o auxílio da alça de platina esterilizada e agitação manual. As suspensões de esporos obtidas foram filtradas em gases estéreis e coletadas em um Bécker. A concentração do inóculo foi ajustada para $2.10^{6}$ conídios $/ \mathrm{mL}$, com auxilio da câmara de Neubauer. Essa suspensão de esporos foi pulverizada em mudas de aroeira-vermelha com quatro meses de idade. Os vasos foram cobertos com saco plástico transparente por 48 horas a fim de manter a umidade sob temperatura em torno de 25 ${ }^{\circ} \mathrm{C}$. As plantas testemunhas foram pulverizadas com água esterilizada. Metodologia adaptada de Botelho (2006).

As mudas foram mantidas na bancada do laboratório em temperatura ambiente, durante o mês de março $\left(\approx 25^{\circ} \mathrm{C}\right.$ ). As avaliações (visualização de sintomas) foram semanais, durante 28 dias. Fragmentos das folhas sintomáticas foram coletados das plântulas 10 dias após a inoculação do fungo e submetidos à desinfestação superficial com álcool (70 \% - 30segundos), hipoclorito de sódio (1\% 30 segundos) e enxágüe triplo em água esterilizada (1 minuto). Após a secagem do material vegetal foi realizada a transferência para placas de Petri, contendo meio de cultura BDA, e mantidos em câmara de incubação, a $25 \pm 2{ }^{\circ} \mathrm{C}$. Depois de sete dias de incubação os fungos que se desenvolveram a partir dos fragmentos das folhas foram identificados.

\section{Resultados e Discussão}

Germinação e sanidade das sementes coletadas

O percentual de germinação das sementes de Schinus terebinthifolius foi baixo, não ultrapassando $16 \%$. Resultados estes que corroboram com os apresentados por Botelho (2006), que relatou índices de germinação de sementes de aroeiravermelha, coletadas na região de Piracicaba - SP, inferiores a $15 \%$. De acordo com dados do IPEF (2006) o percentual de germinação de sementes dessa espécie pode alcançar $60 \%$.

Para Wielewicki et al. (2006) as espécies silvestres, diferentemente das culturas agrícolas, têm ainda alta variabilidade genética, o que resulta em ampla variedade de características morfofisiológicas que determinam o comportamento ecológico de indivíduos da mesma espécie. Além disso, as diferenças edafoclimáticas de cada região e o manejo de coleta e pós-coleta influenciam diretamente na qualidade germinativa das sementes.

No presente trabalho foram identificados nas 
sementes de Schinus terebinthifolius os gêneros Aspergillus, Chaetomium, Cladosporium, Colletotrichum, Penicillium e Pestalotiopsis, sendo que Cladosporium e Pestalotiopsis apresentaram incidências maiores que 50 \% (Figura 1). Botelho
(2006), também identificou esses dois fitopatógenos associados a sementes de aroeira-vermelha, e verificou a influência destes no percentual de germinação.

Figura 1. Percentual de gêneros fúngicos identificados em sementes de $S$. terebinthifolius, coletadas no município de Santa Maria, RS.

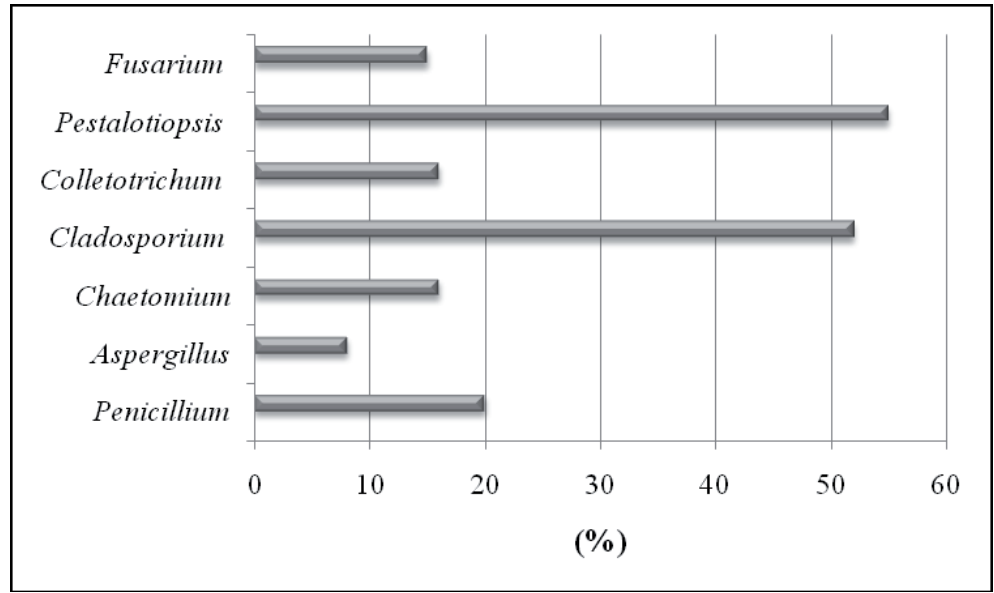

Fonte: Elaboração dos autores.

Medeiros et al. (1992) encontraram 22 gêneros distintos de fungos em sementes de Astronium urundeuva (aroeira), deste total de gêneros, quatro foram comuns aos identificados no presente trabalho, sendo eles: Penicillium, Aspergilus, Cladosporium e Pestalotiopsis.

Faiad, Ramos e Wetzel (2004), estudando diversas espécies florestais, constataram a presença de Cladosporium sp., em alta frequência; segundo esses autores Cladosporium sp. pode ser responsável pela descoloração das sementes e redução na germinação. Martinelli-Seneme et al. (2006) identificaram em Bahuinia variegata diversos fungos comuns aos identificados em S. terebinthifolius, tais como Aspergillus sp., Cladosporium sp., Colletotrichum sp., Fusarium sp. e Penicillium sp., porém estes não exerceram efeito na germinação das sementes.

A contaminação de sementes por patógenos pode ocorrer tanto no campo como nas operações subseqüentes - colheita, secagem e beneficiamento
- afetando a sua qualidade, reduzindo a sua capacidade germinativa e causando tombamento de plântulas recém-emergidas (CARNEIRO, 1990).

\section{Transmissão via semente}

Os fungos identificados no teste de transmissão, associados a sementes de aroeira foram Fusarium sp., Pestalotiopsis sp. e Cladosporium sp. (Figura 2), o percentual de plântulas sintomáticas foi de $22 \%$ e o de sementes não germinadas foi superior a $80 \%$.

Dentre os fungos identificados no teste de sanidade, o gênero Pestalotiopsis foi detectado no teste de transmissão (Figura 2), causando podridão nas sementes de aroeira (Figura 3A) e manchas escuras na plântula emergida (Figura 3B). Há relatos deste fungo causando danos a outras espécies florestais, segundo Dhingra, Maia e Mesquita (2002) Pestalotiopsis sp. foi encontrado em associação com sementes de angico-vermelho (Anadenanthera macrocarpa). Nesta planta o fungo mostrou-se 
altamente patogênico causando podridão na semente e na raiz, redução na altura e definhamento de plântulas sobreviventes. Pestalotiopsis sp. também foi encontrado em sementes de caixeita (Schefflera morototonii) e canafístula (Peltophorum dubium) causando tombamento de plântula (MACHADO et al., 2004).

Figura 2. Percentual de gêneros fúngicos identificados no teste de transmissão em sementes de S. terebinthifolius, coletadas no município de Santa Maria, RS.

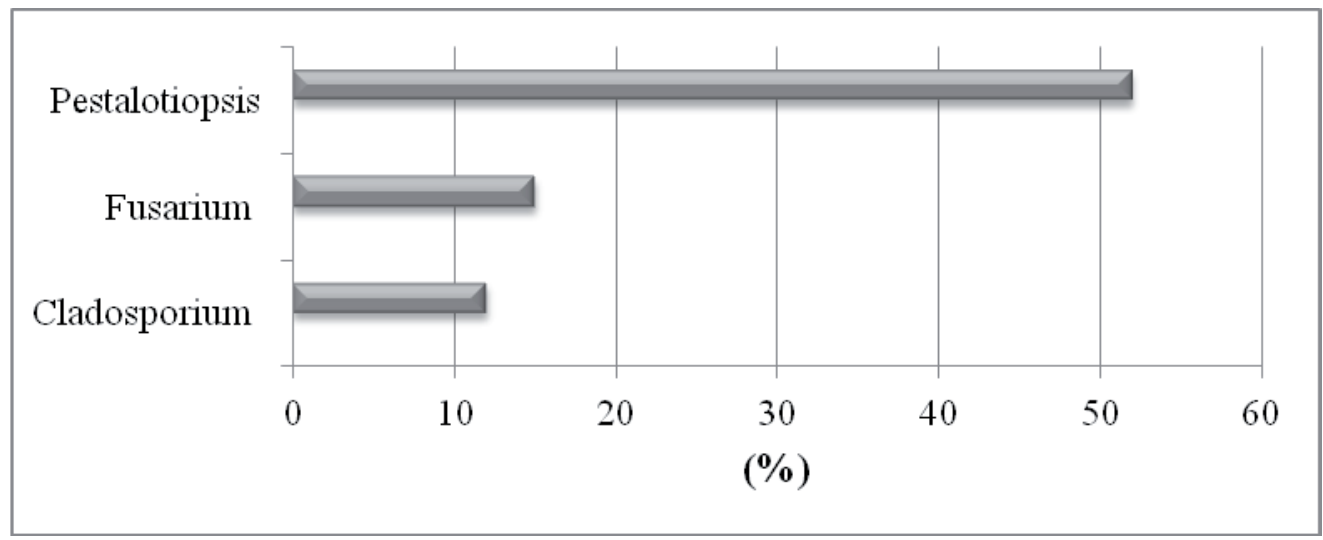

Fonte: Elaboração dos autores.

Figura 3. Material vegetativo de Schinus terebinthifolius infectados com Pestalotiopsis sp. (A) Semente com sinais de infecção pelo fungo; (B) plântula sintomática proveniente do teste de transmissão via semente

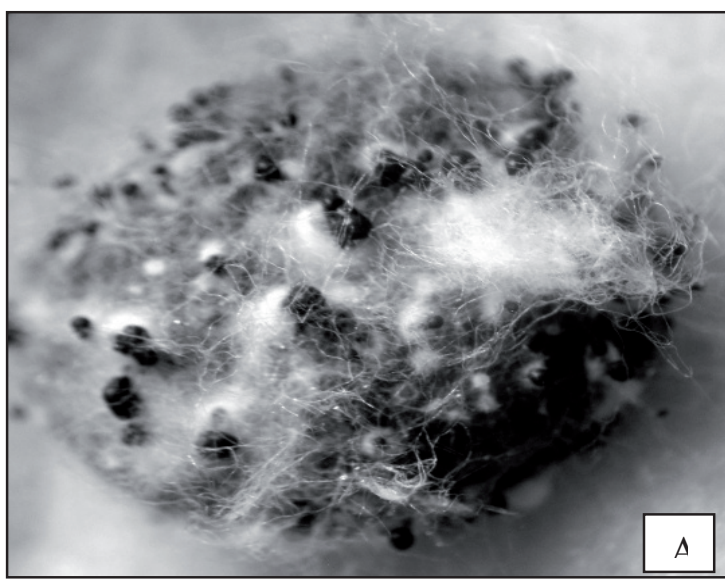

Fonte: Caciara G. Maciel.

\section{Patogenicidade de Pestalotiopsis sp}

Constatou-se que Pestalotiopsis sp. foi patogênico a mudas de Schinus terebinthifolius. Após sua inoculação, observaram-se manchas foliares de formatos irregulares, pequenas e escuras que se desenvolveram causando necrose do tecido

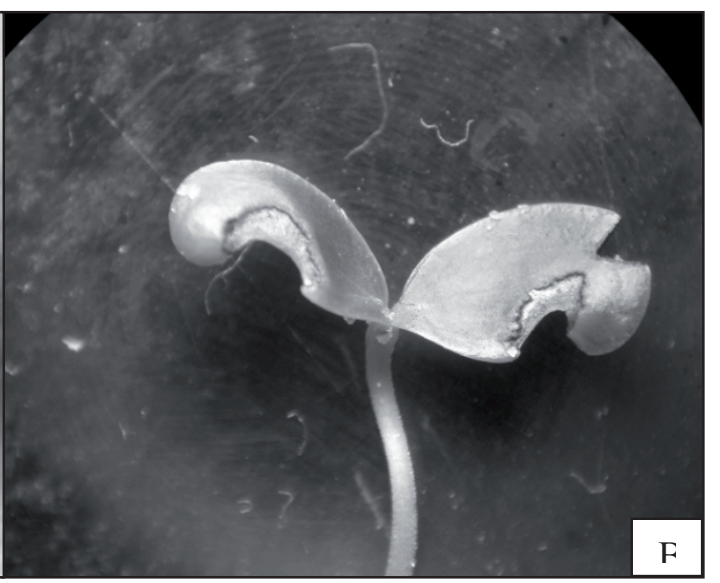

F 
fungos transmitidos por sementes de espécies florestais ainda são escassos. Botelho (2006), relatou a ocorrência de Pestalotiopsis sp. em sementes de $S$. terebinthifolius provenientes da região de São Paulo, este autor verificou a transmissão do fungo para plântulas, bem como a patogenicidade em mudas desta espécie, e os sintomas verificados foram semelhantes aos descritos neste trabalho. Mendes e Muchovej (1991) relataram a presença de Pestalotiopsis maculans em folhas de pau-brasil, causando lesões claras, variando para marrom, no meio ou no bordo do folíolo.

A associação destes patógenos com as sementes, e a possível transmissão para as plântulas, compromete a regeneração natural da espécie, bem como, a qualidade das mudas que são produzidas para comercialização.

Figura 4. Sintoma em mudas de Schinus terebinthifolius após inoculação do fungo Pestalotiopsis sp. (A); crescimento da colônia do fungo em meio de cultura BDA (B); picnídios do fungo vistos em lupa (C) e esporos de Pestalotiopsis sp. observados em microscópio com aumento de 40 x (D). Imagens B, C e D oriundas de folhas com sintomas, após a inoculação de Pestalotiopsis sp.

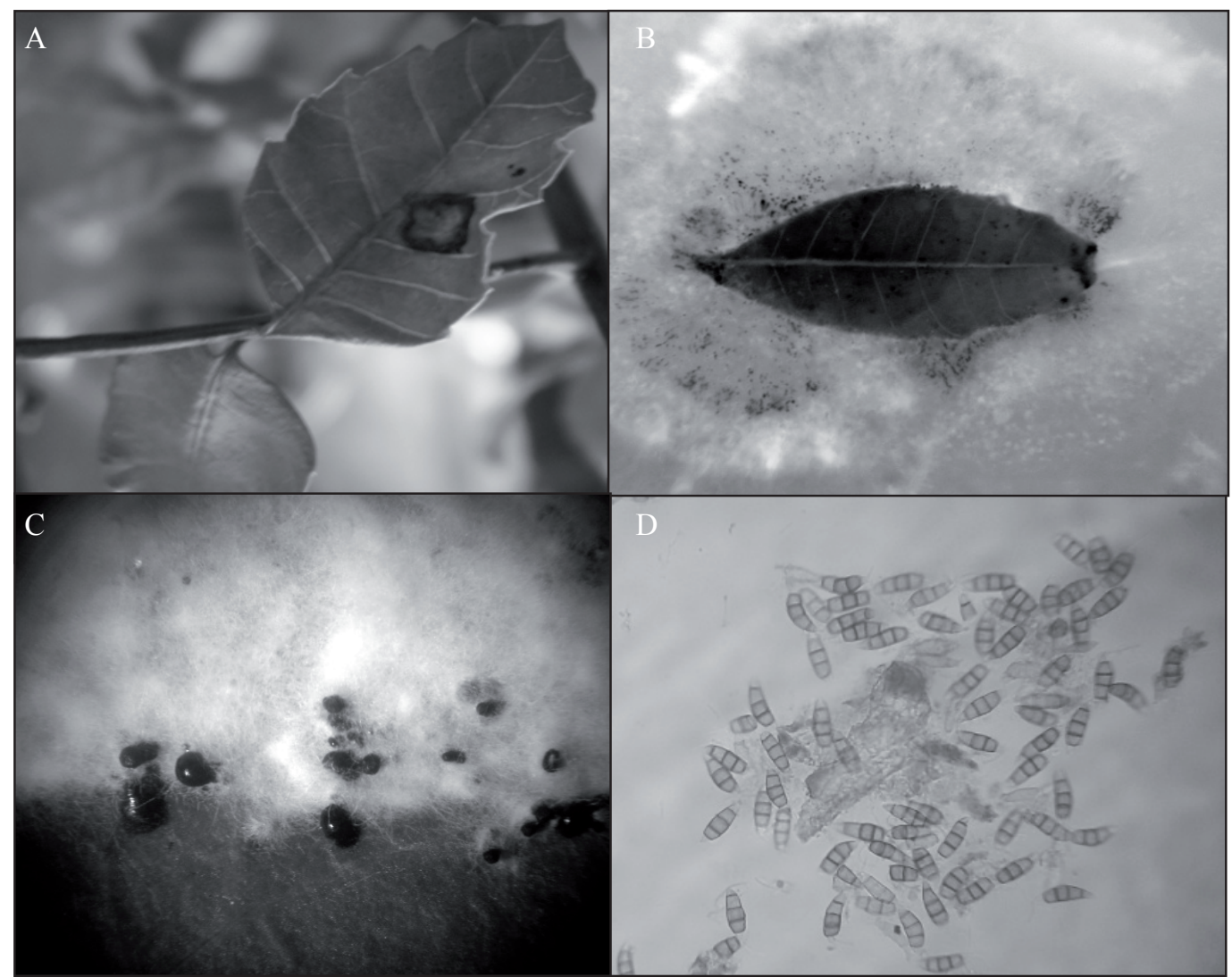

Fonte: Caciara G. Maciel.

\section{Conclusão}

A baixa germinação das sementes de aroeiravermelha $(16 \%)$ está relacionada com a presença de fungos associados à semente. No teste de transmissão identificaram-se os gêneros Fusarium,
Pestalotiopsis e Cladosporium, o percentual de plântulas sintomáticas alcançou $22 \%$.

Pestalotiopsis sp., quando inoculado em mudas de Schinus terebinthifolius mostrou-se patogênico, causando manchas foliares. 


\section{Referências}

BARNETT, H. L.; HUNTER, B. B. Illustred genera of imperfect fungi. $3^{\text {rd }}$ ed. Minneapolis: Burgess Publishing Company, 1972. $241 \mathrm{p}$.

BOTELHO, L. S. Fungos associados às sementes de ipê-amarelo (Tabebuia serratifolia), ipê-roxo (Tabebuia impetiginosa), aroeira-pimenteira (Schinus terebinthifolius) e aroeira-salsa (Schinus molle): incidência, efeitos na germinação, transmissão para plântulas e controle. 2006. Dissertação (Mestrado em Agronomia) - Escola Superior Luiz de Queiroz. Universidade de São Paulo, Piracicaba.

CARNEIRO, J. S. Qualidade sanitária de sementes de espécies florestais em Paraopeba, MG. Fitopatologia Brasileira, Brasília, v. 15, n. 1, p. 75-76, 1990.

DHINGRA, O. D.; MAIA, C. B.; MESQUITA, J. B. Seedborme pathogenic fungi that affect seedling quality of red angico (Anadenanthera macrocarpa) trees in Brazil. Journal of Phytopathology, Saint Paul, v. 150, p. 451-455, 2002.

FAIAD, M. G. R.; RAMOS, V. R.; WETZEL, M. M. V. Patologia de espécies florestais do cerrado. In: SIMPÓSIO BRASILEIRO DE PATOLOGIA DE SEMENTES, 8., 2004, João Pessoa. Palestras e Resumos... João Pessoa, 2004. p. 36-42.

INSTITUTO DE PESQUISA E ESTUDOS FLORESTAIS - IPEF. 2006. Disponível em: <http:// www.ipef. br/identificacao/>. Acesso em: 09 nov. 2011.

LAZAROTTO, M.; MUNIZ, M. F. B.; SANTOS, A. F. dos. Detecção, transmissão, patogenicidade e controle químico de fungos em sementes de paineira (Ceiba speciosa). Summa Phytopathologica, Botucatu, v. 36, n. 2, p. 134-139, abr./jun. 2010.

LEMES, R.; RITTER, C. D.; MORAIS, A. B. B. de. Borboletas (Lepidoptera: Hesperioidea e Papilionoidea) visitantes florais no Jardim Botânico da Universidade Federal de Santa Maria, Santa Maria, RS, Brasil. Biotemas, Florianópolis, v. 21, n. 4, p. 91-98, dez. 2008.

LEONHARDT, C.; TILLMANN, M. A. A.; VILLELA, F. A.; MATTEI, V. L. Maturação fisiológica de sementes de tarumã-de-espinho (Citharexylum montevidense (Spreng.) Moldenke - Verbenaceae) no Jardim Botânico de Porto Alegre, RS. Revista Brasileira de Sementes, Brasília, v. 23, n. 1, p. 100-107, 2001.
LORENZI, H. Árvores brasileiras: manual de identificação e cultivos de plantas arbóreas do Brasil. 2. ed. São Paulo: Nova Odessa, 2002.

MACHADO, A. A.; MUNIZ, M. F. B.; HOPPE, J. M.; CAMARGO, R. Incidência de fungos causadores de "damping off"'em sementes de caixeta ((Schefflera morototonii (Aubl.) Dec.) e canafístula (Peltophorum dubium (Spreng.) Taub.). Fitopatologia Brasileira, Brasília, v. 29, p. 51-51, 2004. Suplemento.

MARTINELLI-SENEME, A.; POSSAMAI, E.; SCHUTA, L. R.; VANZOLINI, S.Germinação e sanidade de sementes de Bauhinia variegata. Revista Árvore, Viçosa, v. 30, n. 5, p. 719-724, set./out. 2006.

MEDEIROS, A. C. S.; MENDES, M. A. S.; FERREIRA, M. A. S. V.; ARAGÃO, F. J. L. Avaliação qualiquantitativa de fungos associados a sementes de aroeira (Astronium urundeuva (FR. ALL.) ENGL.). Revista Brasileira de Sementes, Brasília, v. 14, n. 1, p. 51-55, 1992.

MENDES, M. L.; MUCHOVEJ, J. J. Pestalotiopsis leaf spot of Brazil wood, Caesalpinia echinata. Plant Pathology, London, v. 40, n. 4, p. 635-636, 1991.

REGO, S. S. Germinação, morfologia e sanidade de sementes de Blepharocalyx salicifolius (H.B.K.) Berg e Myrcengenia gerti Landrum - Myrtaceae. 2008. Dissertação (Mestrado em Engenharia Florestal) Universidade Federal do Paraná, Curitiba.

SANTOS, A. F.; MEDEIROS, A. C. S.; SANTANA, D. L. Q. Fungos em sementes de espécies arbóreas da mata Atlântica. Boletim de Pesquisa Florestal, Colombo, n. 42, p. 51-60, jan./jun. 2001.

STRAPASSON, M.; SANTOS, A. F.; MEDEIROS, A. C. $\mathrm{S}$. Fungos Associados às sementes de aroeira-vermelha (Schinus terebinthifolius). Boletim de Pesquisa Florestal, Colombo, n. 45, p. 131-135. jul./dez. 2002.

WIELEWICKI, A. P.; LEONHARDT, C.; SCHLINDWEIN, G.; MEDEIROS, A. C. S. Proposta de padrões de germinação e teor de água para sementes de algumas espécies florestais presentes na região sul do Brasil. Revista Brasileira de Sementes, Brasília, v. 28, n. 3, p. 191-197, 2006. 
